\title{
Quantum Monte Carlo study of the itinerant-localized model of strongly correlated electrons: Spin-spin correlation functions
}

\author{
Ilya Ivantsov, ${ }^{1,2}$ Alvaro Ferraz, ${ }^{3}$ and Evgenii Kochetov ${ }^{2}$ \\ ${ }^{1}$ L. V. Kyrensky Institute of Physics, Siberian Branch of Russian Academy of Sciences, Krasnoyarsk, Russia \\ ${ }^{2}$ Bogoliubov Laboratory of Theoretical Physics, Joint Institute for Nuclear Research, Dubna, Russia \\ ${ }^{3}$ International Institute of Physics - UFRN, Department of Experimental and Theoretical Physics - UFRN, Natal, Brazil \\ (Received 16 August 2016; revised manuscript received 17 October 2016; published 6 December 2016)
}

\begin{abstract}
We perform quantum Monte Carlo simulations of the itinerant-localized periodic Kondo-Heisenberg model for the underdoped cuprates to calculate the associated spin correlation functions. The strong electron correlations are shown to play a key role in the abrupt destruction of the quasi-long-range antiferromagnetic order in the lightly doped regime.
\end{abstract}

DOI: 10.1103/PhysRevB.94.235118

\section{INTRODUCTION}

The aim of this article is to explore the mechanism underlying the abrupt suppression of the long-range antiferromagnetic (AF) order observed in the lightly hole-doped cuprates. As is well known the two-dimensional (2D) undoped quantum AF order exhibits at zero temperature the $\mathrm{AF}$ long-range order (LRO) that is completely destroyed by a surprisingly low doping. It is very reasonable to assume that the strong electron correlations are at work in this case. Technically, the lightly doped regime is pretty hard to address because, precisely under this condition, the constraint of no double electron occupancy (NDO) is fully at work. This implies that, due to the strong on-site Coulomb repulsion, two lattice electrons cannot hop onto one and the same lattice site regardless of their spin projection. Such a local restriction on a structure of the Hilbert space is very hard to implement analytically in a reliable and controlled manner. Alternative slave-particle mean-field theories that treat the local NDO constraint only globally predict a nonphysically large value of the critical doping [1].

The NDO constraint drives the theory into a strongcoupling regime, which calls for proper technical tools. Some progress can be achieved by employing the earlier established mapping of the $t-J$ model of strongly correlated electrons onto the Kondo-Heisenberg model at a dominantly large Kondo coupling [2]. Being a slave-particle theory, such an approach possesses however a few important advantages over the conventional slave-particle theories.

First of all, the strength of electron correlations is now encoded into a single global parameter-a Kondo coupling. Varying its magnitude enables us to get important insights into in what way the strong electron correlations affect the underlying physics. In particular we show that the local NDO constraint is responsible for a rapid destruction of the AF quasi-LRO (QLRO) with doping. If the NDO constraint is ignored the QLRO is restored. The critical hole concentration at which the AF QLRO disappears acquires a reasonably low value.

Additionally, the proposed spin-dopon theory explicitly takes into account the dual nature of the constrained lattice electrons. In the underdoped cuprates, one striking feature is the simultaneous localized and itinerant nature of the lattice electrons. Such a duality appears as an explicit manifestation of the local Mott physics and is shown to be a direct consequence of the local NDO constraint.

Moreover, the itinerant-localized model provides a convenient new set of coordinates well suited for numerical simulations. Specifically, one can vary the strength of the electron correlations by simply varying a single global parameterthe (Kondo) coupling between the itinerant and localized electrons. In particular, classical Monte Carlo simulations for large clusters have been successfully used in studying the electron spin correlations in the full Ising version of the $2 \mathrm{D}$ $t$ - $J$ model in the spin-dopon representation [3]. It has been shown that the AF LRO disappears already at the doping of the order of a few percent. It has also been demonstrated that the NDO constraint is responsible for the smearing out of the magnetic order. However, these results were obtained within a simplified model with the transverse components of the on-site electron spin being self-consistently neglected.

In the present paper, we apply the quantum Monte Carlo (QMC) simulations to explore the quantum spin dynamics of the underdoped cuprates within the standard SU(2) invariant 2D $t-J$ model. We intend to explore the issue as to whether or not the NDO constraint still plays a dominant role in the disruption of the magnetic order in the lightly doped regime. One should however keep in mind that the QMC method restricts us to dealing with finite temperatures and finite lattice clusters. As a result, this approach cannot capture a true LRO in two dimensions. Since the AF correlation length remains finite, we consider a finite-size system away from the critical point. A full theory of such systems is not available yet. However, at sufficiently low doping, the correlation length is much larger than a characteristic cluster size. This manifests itself as a QLRO. What is important is that the QMC method enables us to observe a rapid destruction of the QLRO with increasing doping and the formation of the short-range order (SRO) instead. We explicitly demonstrate that the local NDO constraint plays a dominant role in destroying the magnetic order at finite doping in the standard $t-J$ model.

\section{MODEL}

To start with, let us briefly review the Kondo-Heisenberg model approach to strongly correlated electron systems. The canonical $t-J$ model Hamiltonian of strongly correlated 
electrons reads

$$
H_{t-J}=-\sum_{i j \sigma} t_{i j} \tilde{c}_{i \sigma}^{\dagger} \tilde{c}_{j \sigma}+J \sum_{i j}\left(\vec{Q}_{i} \cdot \vec{Q}_{j}-\frac{1}{4} \tilde{n}_{i} \tilde{n}_{j}\right),
$$

where $\tilde{c}_{i \sigma}=c_{i \sigma}\left(1-n_{i,-\sigma}\right)$ is the projected electron operator, $\vec{Q}_{i}=\sum_{\sigma, \sigma^{\prime}} \tilde{c}_{i \sigma}^{\dagger} \vec{\tau}_{\sigma \sigma^{\prime}} \tilde{c}_{i \sigma^{\prime}}$ is the electron spin operator, $\tilde{n}_{i}=$ $n_{i \uparrow}+n_{i \downarrow}-2 n_{i \uparrow} n_{i \downarrow}$, and $\vec{\tau}$ is the Pauli vector, $\vec{\tau}^{2}=3 / 4$. In the underdoped cuprates, one striking feature is a simultaneous display of both the localized nature and the itinerant nature of the lattice electrons, $\tilde{c}_{i \sigma}$. To take both these aspects of the constrained electrons into consideration, on equal footing, Ribeiro and Wen proposed a slave-particle spin-dopon representation of the projected electron operators in the enlarged Hilbert space [4]:

$$
\tilde{c}_{i}^{\dagger}=\frac{1}{\sqrt{2}}\left(\frac{1}{2}-2 \vec{S}_{i} \cdot \vec{\tau}\right) \tilde{d}_{i} .
$$

In this framework, the localized electron is represented by the lattice spin $\vec{S} \in s u(2)$ whereas the doped hole (dopon) is described by the projected hole operator, $\tilde{d}_{i \sigma}=d_{i \sigma}\left(1-n_{i-\sigma}^{d}\right)$. Here $\tilde{c}^{\dagger}=\left(\tilde{c}_{\uparrow}^{\dagger}, \tilde{c}_{\downarrow}^{\dagger}\right)^{t}$ and $\tilde{d}=\left(\tilde{d}_{\uparrow}, \tilde{d}_{\downarrow}\right)^{t}$.

The physical content of the spin-dopon representation (2) can be clarified as follows. First of all, we represent the spin degrees of freedom in terms of chargeless fermions (spinons), $f_{\sigma}$ :

$$
S=\sum_{\sigma, \sigma} f_{\sigma^{\prime}}^{\dagger} \vec{\tau}_{\sigma^{\prime} \sigma} f_{\sigma}, \quad \sum_{\sigma} f_{\sigma}^{\dagger} f_{\sigma}=1
$$

Following this, we introduce the operator [2]

$$
D=\frac{f_{\uparrow} \tilde{d}_{\downarrow}-f_{\downarrow} \tilde{d}_{\uparrow}}{\sqrt{2}},
$$

which destroys the on-site spin-dopon singlet state (holon). The physical electron operator (2) then reduces to the spinonholon decomposition:

$$
\tilde{c}_{\sigma}^{\dagger}=f_{\sigma}^{\dagger} D
$$

This equation appears as a slave-boson representation of the constrained electron operator in terms of the itinerant and localized degrees of freedom with the boson being a composite state. The itinerant boson (holon) appears as a charged spinondopon singlet and it corresponds to a hopping vacancy. The localized lattice spin is represented by a chargeless spinon state that transforms as an $\mathrm{SU}(2)$ spinor.

The physical on-site Hilbert space is a $3 \mathrm{D}$ one that comprises spin-up states, spin-down states, and a vacancy. In terms of the projected electron operators, the NDO constraint to single out the physical Hilbert space takes the form

$$
\sum_{\sigma}\left(\tilde{c}_{i \sigma}^{\dagger} \tilde{c}_{i \sigma}\right)+\tilde{c}_{i \sigma} \tilde{c}_{i \sigma}^{\dagger}=1 .
$$

Only under this condition are the projected electron operators isomorphic to the Hubbard operators. Within the spin-dopon representation, the NDO reduces to a Kondo-type interaction constraint [2],

$$
\vec{S}_{i} \cdot \vec{s}_{i}+\frac{3}{4}\left(\tilde{d}_{i \uparrow}^{\dagger} \tilde{d}_{i \uparrow}+\tilde{d}_{i \downarrow}^{\dagger} \tilde{d}_{i \downarrow}\right)=0,
$$

with $\vec{s}_{i}=\sum_{\sigma^{\prime}, \sigma} \tilde{d}_{i \sigma^{\prime}}^{\dagger} \vec{\tau}_{\sigma^{\prime} \sigma} \tilde{d}_{i \sigma}$ being the dopon spin operator. Equivalently, Eq. (5) can be written in the form $D_{i}^{\dagger} D_{i}=\tilde{n}_{i}^{d}$.

At strong coupling $(\lambda \gg t)$, the original $t-J$ model $(1)$ is shown to be equivalent to the lattice Kondo-Heisenberg-type model [2]:

$$
\begin{aligned}
H_{t-J}= & \sum_{i j \sigma} 2 t_{i j} d_{i \sigma}^{\dagger} d_{j \sigma}+J \sum_{i j} \vec{S}_{i}\left(1-n_{i}^{d}\right) \cdot \vec{S}_{j}\left(1-n_{j}^{d}\right) \\
& +\lambda \sum_{i}\left(\vec{S}_{i} \cdot \vec{s}_{i}+\frac{3}{4} n_{i}^{d}\right), \quad \lambda \rightarrow+\infty,
\end{aligned}
$$

where we have dropped the "tilde" sign of the dopon operators, as it becomes irrelevant in the presence of the NDO constraint. The unphysical doubly occupied electron states are separated from the physical sector by an energy gap of $\sim \lambda$. In the $\lambda \rightarrow+\infty$ limit, i.e., in the limit in which $\lambda$ is much larger than any other existing energy scale in the problem, those states are automatically excluded from the Hilbert space. Despite the global character of the parameter $\lambda$, it enforces the NDO constraint locally due to the fact that the on-site physical Hilbert subspace corresponds to zero eigenvalues of the constraint, whereas the nonphysical subspace is spanned by the eigenvectors with strictly positive eigenvalues. In one dimension, Eq. (6) reproduces the well-known exact results for the $t-J$ model [5] (see also the Appendix).

Close to half filling, where the density of doped holes is small $\delta:=\left\langle n_{i}^{d}\right\rangle \ll 1$, it suffices to change $J \rightarrow \tilde{J}=J(1-$ $\delta)^{2}$. The spin-dopon representation of the $t-J$ Hamiltonian for the underdoped cuprates then reduces to the KondoHeisenberg lattice model at a dominantly large Kondo coupling [2],

$$
H_{t-J}=\sum_{i j \sigma} t_{i j}^{\mathrm{eff}} d_{i \sigma}^{\dagger} d_{j \sigma}+\tilde{J} \sum_{i j}\left(\vec{S}_{i} \cdot \vec{S}_{j}-\frac{1}{4}\right)+\lambda \sum_{i} \vec{S}_{i} \cdot \vec{s}_{i},
$$

where $t_{i j}^{\text {eff }}=2 t_{i j}+(3 \lambda / 4-\mu) \delta_{i j}$ and $\lambda \gg t, J$.

In the spin-dopon representation (7), the on-site Hilbert space is spanned by the vectors $|\sigma a\rangle$, with $\sigma=\uparrow, \downarrow$ labeling the lattice spin projection and $a=0, \uparrow, \downarrow$ labeling the dopon state. Explicitly they are numerated by an integer $p=$ $1,2, \ldots, 6$ as given in Table I.

Any on-site operator $A$ can then be identically written in the form $A_{i}=\sum_{p q}\left\langle p\left|A_{i}\right| q\right\rangle X_{i}^{p q}$, where $X^{p q}:=|p\rangle\langle q|$.

Since we are interested in the large $\lambda$ limit, it seems appropriate to separate the Hamiltonian in the following way: $H_{t-J}=H_{\lambda}+H_{z}+H_{\text {int }}$, where

$$
\begin{aligned}
& H_{\lambda}=\lambda \sum_{i} \sum_{p q}\left(\frac{3}{4}\left\langle p\left|n_{i}^{d}\right| q\right\rangle+\left\langle p\left|\vec{S}_{i} \cdot \vec{s}_{i}\right| q\right\rangle\right) X_{i}^{p q}, \\
& H_{z}=\tilde{J} \sum_{i j} \sum_{p q}\left\langle p\left|S_{i}^{z}\right| q\right\rangle\left\langle m\left|S_{j}^{z}\right| n\right\rangle X_{i}^{p q} X_{j}^{m n},
\end{aligned}
$$

TABLE I. The basis states.

\begin{tabular}{lccccc}
\hline \hline$|1\rangle$ & $|2\rangle$ & $|3\rangle$ & $|4\rangle$ & $|5\rangle$ & $|6\rangle$ \\
\hline$|\uparrow \uparrow\rangle$ & $|\uparrow 0\rangle$ & $|\uparrow \downarrow\rangle$ & $|\downarrow \uparrow\rangle$ & $|\downarrow 0\rangle$ & $|\downarrow \downarrow\rangle$ \\
\hline \hline
\end{tabular}


TABLE II. The new basis states.

\begin{tabular}{cccccc}
\hline \hline$|1\rangle$ & $|2\rangle$ & $|3\rangle$ & $|4\rangle$ & $|5\rangle$ & $|6\rangle$ \\
\hline$|\uparrow \uparrow\rangle$ & $|\uparrow 0\rangle$ & $\frac{|\uparrow \downarrow\rangle-|\downarrow \uparrow\rangle}{\sqrt{2}}$ & $\frac{|\uparrow \downarrow\rangle+|\downarrow \uparrow\rangle}{\sqrt{2}}$ & $|\downarrow 0\rangle$ & $|\downarrow \downarrow\rangle$ \\
\hline \hline
\end{tabular}

$$
\begin{aligned}
H_{\mathrm{int}}= & \sum_{i j \sigma} \sum_{\mathrm{pqmn}} 2 t_{i j}\left\langle p\left|d_{i \sigma}^{\dagger}\right| q\right\rangle\left\langle m\left|d_{j \sigma}\right| n\right\rangle X_{i}^{p q} X_{j}^{m n} \\
& +\frac{\tilde{J}}{2} \sum_{i j} \sum_{\mathrm{pqmn}}\left\langle p\left|S_{i}^{+}\right| q\right\rangle\left\langle m\left|S_{j}^{-}\right| n\right\rangle X_{i}^{p q} X_{j}^{m n}+\text { H.c. }
\end{aligned}
$$

In this basis $H_{\lambda}$ takes on a nondiagonal form:

$$
H_{\lambda}=\lambda \sum_{i}\left[X_{i}^{11}+X_{i}^{66}+\frac{1}{2}\left(X_{i}^{33}+X_{i}^{34}+X_{i}^{43}+X_{i}^{44}\right)\right] \text {. }
$$

This form is inconvenient for numerical purposes, however. In the large $\lambda$ limit, the probability of the updating procedure involving $\lambda$ becomes much higher than the others. This leads to a crucial slowdown of the calculations. To get around this problem, it is more convenient to go over to the basis constructed out of the eigenstates of $H_{\lambda}$ as given in Table II.

In this case, the $H_{\lambda}$ becomes diagonal in the $(p, q)$ representation:

$$
H_{\lambda}=\lambda \sum_{i}\left(X_{i}^{11}+X_{i}^{66}+X_{i}^{44}\right)
$$

where the unphysical spin triplet states $|p\rangle, p=1,4,6$, enter with eigenvalue $\lambda$. The physical vectors $|p\rangle, p=2,3,5$, that describe the vacancies and lattice spins correspond to zero eigenvalues of $H_{\lambda}$. Due to the fact that the statistical weights of configurations with states $|p\rangle, p=1,4,6$, are proportional to $e^{-\beta \lambda}$, we can exclude these states from calculation, provided $\lambda$ is large enough. From now on all the states denoted by $|p\rangle$ correspond to those from Table II.

\section{METHOD}

In our calculations, we use the continuous time worldline (CTWL) QMC method. Following an appropriate route [6] the algorithm is modified by adding "worms" in the representation of the $X$ operators, which corresponds to the addition of a fictitious term to the Hamiltonian:

$$
H_{v}=\sum_{i p q} v_{p q}\left(X_{i}^{m n}+X_{i}^{n m}\right),
$$

where $v_{p q}$ is a set of fictitious amplitudes satisfying $v_{p p}=0$ and $v_{p q}=v_{q p}$ which are chosen to improve the convergence. These terms are included in the nondiagonal part corresponding to the existence of a worm in the configuration. Since all measurements occur in the absence of the worms, they do not contribute to the final result. This update allows us to make calculations more effectively by adding the fictitious configurations to the true ones. In particular, one is able to run calculations in the grand canonical ensemble keeping at the same time the total number of particles under control.
The QMC method is based on the representation of the partition function in the interaction picture [7]:

$$
e^{-\beta H_{t-J}}=e^{-\beta H_{0}} T_{\tau}\left[\exp \left(-\int_{0}^{\beta} H_{1}(\tau) d \tau\right)\right],
$$

where $T_{\tau}$ denotes the $\tau$-ordering operator, and

$$
\begin{aligned}
H_{t-J} & =H_{0}+H_{1}, \\
H_{0} & =H_{\lambda}+H_{z}^{\text {diag }}, \\
H_{1} & =H_{\text {int }}+H_{v}+H_{z}^{\text {nondiag }} .
\end{aligned}
$$

The partition function expansion takes the form

$$
\begin{aligned}
Z= & S p\left[e ^ { - \beta H _ { 0 } } \left(1-\int_{0}^{\beta} H_{1}(\tau) d \tau\right.\right. \\
& \left.\left.+\int_{0}^{\beta} \int_{0}^{\tau_{1}} H_{1}\left(\tau_{1}\right) H_{1}\left(\tau_{2}\right) d \tau_{1} d \tau_{2}\right)-\ldots\right],
\end{aligned}
$$

where

$$
H_{1}(\tau):=e^{-\tau H_{0}} H_{1} e^{\tau H_{0}} .
$$

The representation of Eqs. (12)-(14) allows us to consider the cases of large and small $\lambda$ on equal footing. In either case, $H_{0}$ represents the leading contribution to the partition function. In particular, a quasi-long-range order is restored at small $\lambda$, in which case the $S^{z} S^{z}$ interaction term in $H_{0}$ becomes of major importance.

In the CTWL method, the expansion of the partition function comes in the form of the worldline states in imaginary time. It is convenient to rewrite the Hamiltonian in a form suitable for this method:

$$
\begin{aligned}
H_{t-J}= & \sum_{i, p} E_{p} X_{i}^{p p}+\sum_{i j, p q} V_{i j}^{p q} X_{i}^{p p} X_{j}^{q q} \\
& +\sum_{i j, p q r s} T_{i j}^{p q r s} X_{i}^{p q} X_{j}^{r s}+\sum_{i, p q} v_{p q}\left(X_{i}^{p q}+X_{i}^{q p}\right),
\end{aligned}
$$

where $E_{p}$ are the eigenvalues of the one-site part of the Hamiltonian (10), whereas $V_{i j}^{p q}$ are the energies of the diagonal interaction between the states $|p\rangle$ and $|q\rangle$ on sites $i$ and $j$, respectively. In this form, for each segment of the horizontal line that lies between $\tau_{1}$ and $\tau_{2}$ in state $|p\rangle$, there corresponds the multiplier $e^{-\beta\left(E_{p}+V\right) d \tau}$, where $V$ is the average energy of the diagonal interaction over all neighboring segments and $d \tau=\tau_{2}-\tau_{1}$ is the imaginary time interval of the segment.

To each kink (a segment of the vertical line in Fig. 1) between sites $i$ and $j$ there corresponds the multiplier $T_{i j}^{p q m n} X_{i}^{p q} X_{j}^{m n}$, where $T_{i j}^{p q m n}$ are the energies of the nondiagonal interaction that corresponds to the state change. Also, with respect to the worm algorithm, to each discontinuity there corresponds the multiplier $v_{p q} X_{i}^{p q}$ that represents the worm boundary, where $v_{p q}$ is the above-introduced fictitious energy.

Figure 1 shows the fragments of a typical configuration occurring during the simulation. The update of the configurations occurs according to the Metropolis algorithm [8] involving a finite number of updating procedures. Those updating procedures are described in detail elsewhere [6]. However, to increase the convergence as well as to ensure the ergodicity of the algorithm, this must be also accompanied by certain additional prescriptions [9]. 
(a)

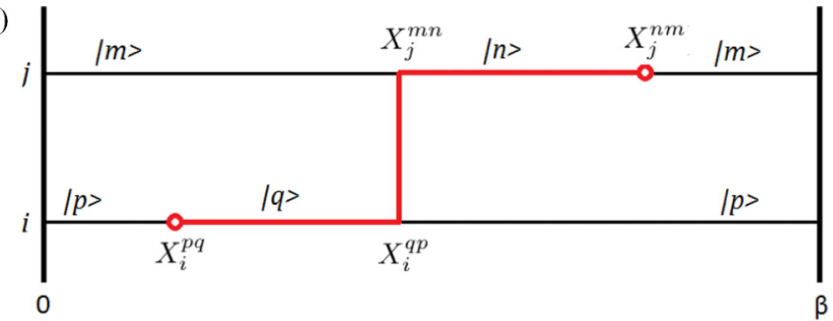

(b)

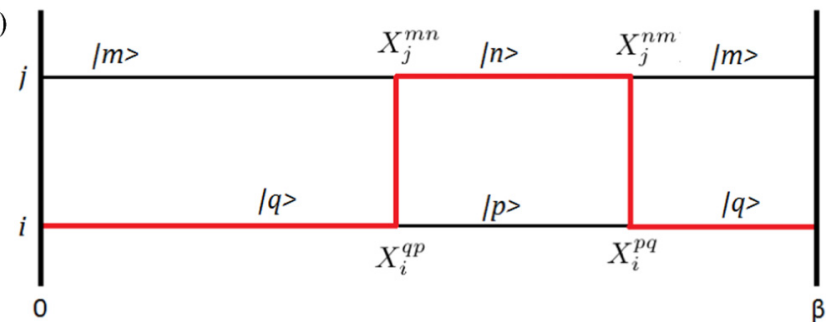

FIG. 1. The fragments of typical configurations. Panels show configurations with (a) and without (b) a worm. In panel (a), the red color indicates the worldline of the worm; in panel (b), the red color indicates the loop formed by the worm closing.

The observables are measured in the following way:

$$
\langle A\rangle:=\frac{\sum_{M C}\left\langle p\left|A e^{-\beta H}\right| p\right\rangle}{\sum_{M C} 1},
$$

where $A$ is some operator. Unfortunately in the fermion system we are faced with the sign problem. This problem is connected with the appearance of the negative statistical weights during the calculation:

$$
\langle A\rangle=\frac{\sum_{M C}\left\langle p\left|A e^{-\beta H}\right| p\right\rangle \operatorname{sign}(W)}{\sum_{M C} \operatorname{sign}(W)} .
$$

As a result, the errors increase exponentially with decreasing temperature, which rules out an acceptable accuracy at low temperatures. Finally, the adopted algorithm goes through the following steps.

(i) An initial configuration is generated. In fact, the initial configuration selection has no impact on the final result. All possible impacts of this choice are eliminated by thermalization.

(ii) Possible updating procedures are chosen randomly. The probabilities of the procedures are not constants but rather depend on the worms and kinks presented in the current configuration. It should also be noted that every procedure has its own inverse. The probabilities of such procedures must be chosen in accordance with the direct ones.

(iii) The site $i$ and times $\tau_{1}$ and $\tau_{2}$ are chosen according to the procedure. The site $i$ is chosen directly in the case of the worm-dependent procedure and randomly otherwise. The moments of time are calculated in accordance with the probability density calculated for each case.

(iv) The probability $W$ of accepting a new configuration is calculated. If $W>R$, where $R$ is a random number from the interval $[0,1]$, the new configuration is accepted. If the updating procedure is interrupted due to the impossibility of the updates, it corresponds to the case $W=0$. However, such interruptions are part of the statistics and they occur in accordance with the detailed balance principle.

(v) In the case where the system has no worms regardless of the accepting of the new configuration, the statistics is supplemented by the new data and the procedure goes back to step (ii), otherwise the procedure goes to step (ii) without the supplementing of data.

Furthermore, by the fact that the CPU time depends linearly on the lattice size and the average sign remains large enough, which helps to keep errors in acceptable limits, the simulation can be made at a relatively large lattice size. All numerical results were obtained for a $20 \times 20$ lattice cluster with the periodic boundary condition. However, this size is not ideal to ensure that the finite-size effects have no significant effects on the result. To make these effects totally negligible the size of the lattice cluster should be extended to at least $30 \times 30$.

\section{RESULTS}

To estimate the dependence of the AF order from the doping level we compute the spin-spin correlation function $g(r)$ for the physical electron operators. This is calculated with
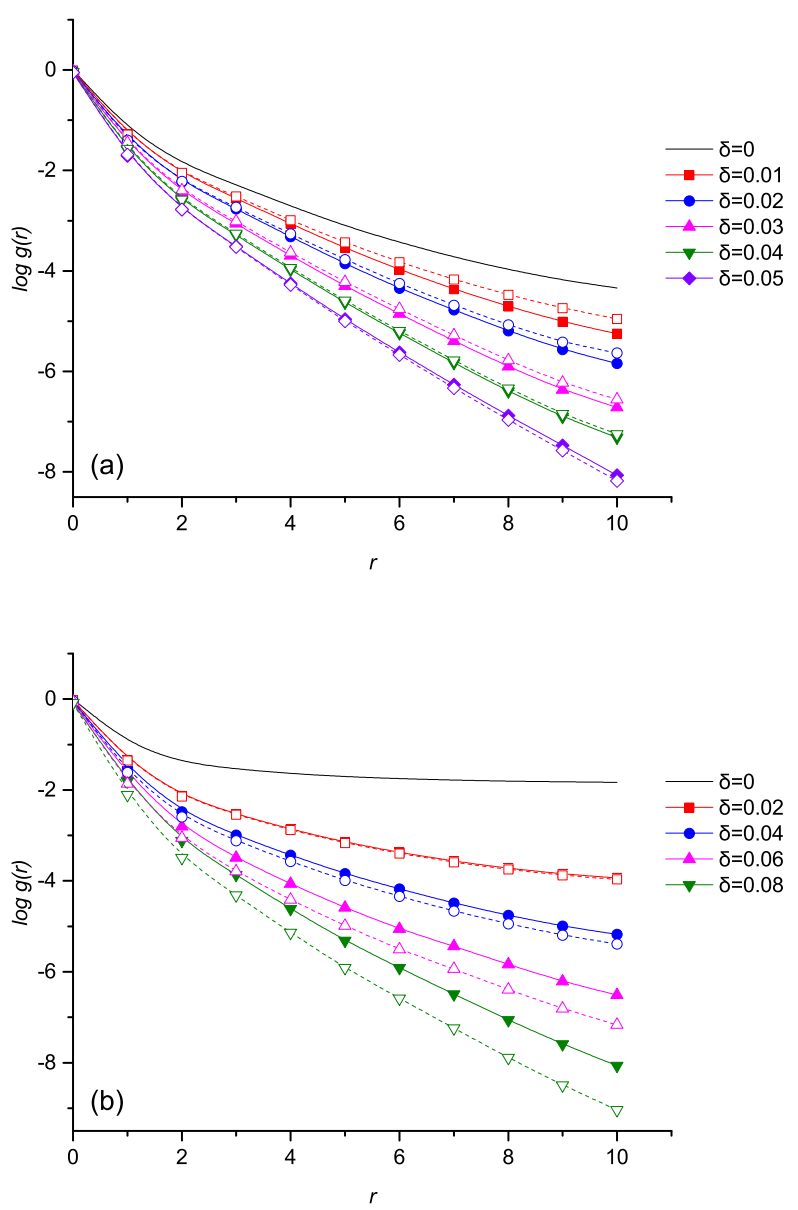

FIG. 2. Panels (a) and (b) show $\log g(r)$ for $J=0.2 t$ (a) and $J=$ $0.4 t$ (b) with $T=0.1 t$. Solid (dashed) lines show results obtained for $t^{\prime}=t^{\prime \prime}=0\left(t^{\prime}=-0.27 t, t^{\prime \prime}=0.2 t\right)$, respectively. 
$\sum_{i} S_{i}^{z}=\sum_{i} s_{i}^{z}=0$ and a fixed number of dopons, $\delta$ :

$$
\begin{aligned}
g(r)= & 4 \Delta^{-1}(r) \sum_{i j} \sum_{p q}\left\langle p\left|S_{i}^{z}+s_{i}^{z}\right| p\right\rangle\left\langle q\left|S_{j}^{z}+s_{j}^{z}\right| q\right\rangle \\
& \times\left\langle X_{i}^{p p} X_{j}^{q q}\right\rangle e^{i \mathbf{K} \cdot\left(\mathbf{R}_{i}-\mathbf{R}_{j}\right)} \bar{\delta}\left(r-\left|\mathbf{R}_{i}-\mathbf{R}_{j}\right|\right),
\end{aligned}
$$

where $\mathbf{K}=(\pi, \pi), \mathbf{R}_{i}$ is the radius-vector of the site $i, \Delta(r)=$ $\sum_{i j} \bar{\delta}\left(r-\left|\mathbf{R}_{i}-\mathbf{R}_{j}\right|\right)$, and

$$
\bar{\delta}(x)= \begin{cases}1 & \text { if }|x| \leqslant 0.5 a \\ 0 & \text { otherwise }\end{cases}
$$

with $a$ being the lattice constant and $\langle\cdots\rangle$ means an average over the spin configurations generated in the QMC run. In all the figures showing $g(r)$ we use the logarithmic scale for the vertical axis. Therefore, for the LRO, the QLRO, and the SRO, the $g(r)$ should be represented asymptotically by a constant, a logarithmic function, and a straight line, respectively.

Figure 2 displays the electron spin-spin correlators for the different doping levels. The critical hole concentration varies from around $\delta_{c}=0.05$ at $J=0.2 t$ to $\delta_{c}=0.08$ at $J=0.4 t$. Due to the finite lattice size as well as to finite temperature effects a true long-range AF order manifests itself as a QLRO even at a very small doping. The suppression of the true LRO corresponds to the destruction of the QLRO due to the
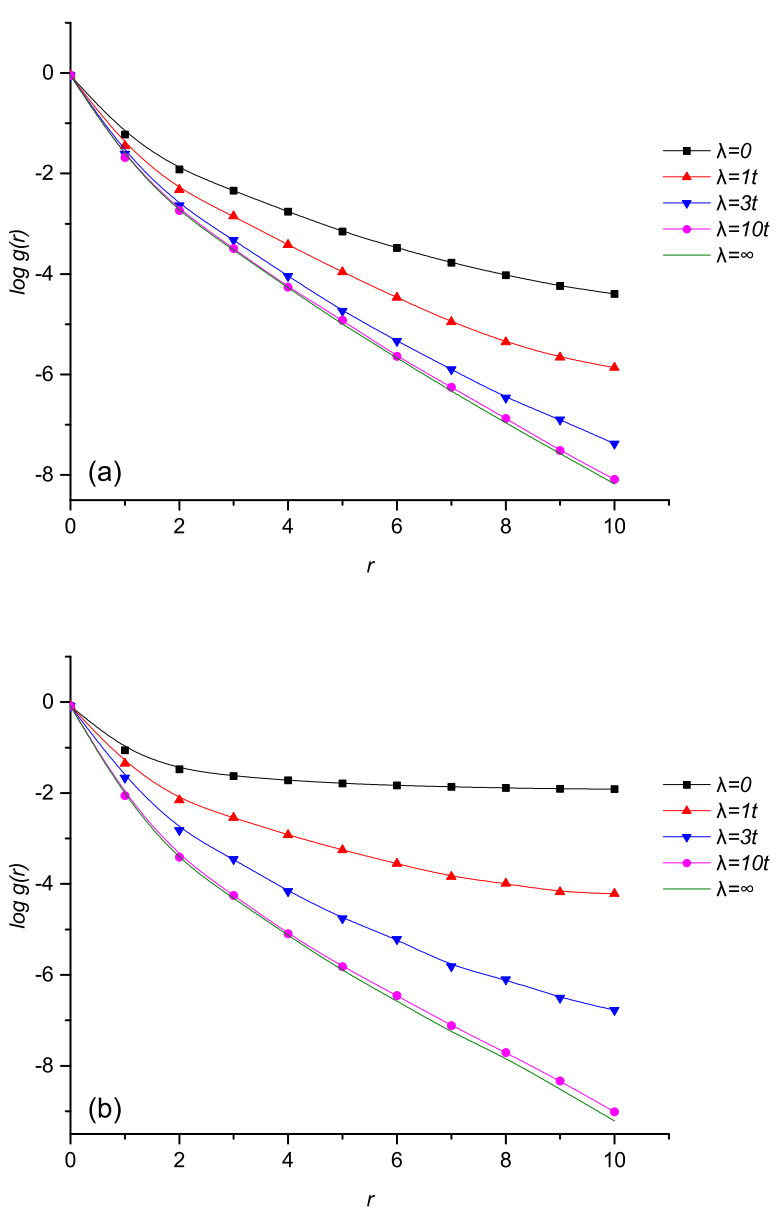

FIG. 3. Panels (a) and (b) show $\log g(r)$ for $J=0.2 t$ and $\delta=0.05$ (a) and $J=0.4 t$ and $\delta=0.08$ (b) with $T=0.1 t$ and $t^{\prime}=-0.27 t, t^{\prime \prime}=0.2 t$ emergence of the short-range AF correlations. The obtained values of the critical hole concentrations do not necessarily coincide with the true ones to be computed at zero temperature in the thermodynamic limit. However their magnitudes are reasonably small.

In Fig. 3 we report the spin-spin correlators, $\log g(r)$, for $J=0.2 t$ and $J=0.4 t$ at $\delta_{c}=0.05$ and $\delta_{c}=0.08$ for different values of $\lambda$, respectively. It is clearly seen that the QLRO is restored as $\lambda$ decreases. The local NDO constraint plays the dominating role in the destruction of the long-range AF state. At $\lambda>10 t$, the spin-spin correlation functions become almost identical to each other. This indicates that finite but large enough values of $\lambda$ already provide a reliable description of the existing strong correlations. In this limit, the high- and lowenergy itinerant fermions cannot be separated out and this is another manifestation of the duality of the lattice electron nature.

It should be noticed that the physical meanings of the Kondo coupling $\lambda$ within the conventional phenomenological spinfermion model [10] and in our Eq. (7) are completely different from each other. In the former case, it represents a spin-densitywave gap that can evolve from small to large values. In our theory, the only meaningful value of the Kondo coupling is that of $\lambda \gg t$ to take proper care of the NDO constraint.

\section{CONCLUSION}

To conclude, we investigate the spin-spin correlation functions in the underdoped $t-J$ model numerically by employing quantum Monte Carlo simulations on finite clusters. Our main conclusion is that it is the local NDO constraint that is behind the rapid suppression of the AF QLRO at a surprisingly small doping level. In contrast, any mean-field global treatment of the local NDO results in unphysically large values of the critical hole concentration.

The itinerant-localized duality of the lattice electrons offers the following explanation of the rapid destruction of the magnetic order by strong correlations. The localized individual lattice spins become less correlated with each other due to the competition between the AF correlations (the characteristic energy scale $\sim J)$ and the Kondo screening $(\sim \lambda)$ of the local spin moments by the conduction dopons. The screening breaks the AF bonds. In the case where a double occupancy is allowed, this breaking is not very efficient, since it is then induced by a small (in this regime) spin-dopon interaction $\lambda$.

As $\lambda$ increases, the screening becomes more effective. Since $1 / J \gg 1 / t$, the hole dynamics is much faster than the spin one. The broken AF bonds recover themselves at a much slower rate than the breaking occurs. As a result, even a small amount of fast-moving dopons (holes) turns out to be, at a large enough $\lambda$, sufficient to completely destroy the AF LRO.

A further possible application of the present approach might be to theoretically explore an experimentally observed instability towards a formation of a charge order in the pseudogap phase at $\delta \approx 0.1$ There is strong evidence that the observed charge order is due to strong electron correlations [11]. The spin-dopon representation of the $t-J$ model provides a natural framework to address this problem. By varying $\lambda$, we would be able to vary the strength of the correlations to explicitly explore the impact of the NDO on the charge order formation. This is already in progress and results will be presented elsewhere. 


\section{APPENDIX}

For the 1D $t-J$ model, the two leading terms of the groundstate energy expansion in powers of $J / t \ll 1$ are known explicitly. In this appendix, we show that the spin-dopon model (6) produces exactly the same result.

As the sign of $t$ is irrelevant, we can fix the Hamiltonian (6) in one dimension to take the form

$$
H_{t-J}=H_{J=0}+H_{\mathrm{int}},
$$

where

$$
\begin{aligned}
H_{J=0}= & : H_{0}=-2 t \sum_{i j \sigma} d_{i \sigma}^{\dagger} d_{j \sigma} \\
& +\frac{3 \lambda}{4} \sum_{i \sigma} d_{i \sigma}^{\dagger} d_{i \sigma}+\lambda \sum_{i} \vec{S}_{i} \cdot \vec{s}_{i}, \quad t>0,
\end{aligned}
$$

and

$$
H_{\mathrm{int}}=J \sum_{i j} \vec{S}_{i} \vec{S}_{j}\left(1-n_{i}^{d}\right)\left(1-n_{j}^{d}\right)
$$

The limit $\lambda \rightarrow \infty$ reduces the local Hilbert space to that comprising a lattice spin-up state $|\uparrow\rangle_{i}=|\uparrow 0\rangle_{i}$, a spin-down state $|\uparrow\rangle_{i}=|\uparrow 0\rangle_{i}$, and a vacancy state $|0\rangle_{i}=\frac{|\uparrow \downarrow\rangle_{i}-|\downarrow \uparrow\rangle_{i}}{\sqrt{2}}$. We define the basis of the one-vacancy states as

$$
|i,\{\sigma\}\rangle=\left|\sigma_{1} \sigma_{2} \ldots 0_{i} \ldots \sigma_{N}\right\rangle
$$

where $\sigma_{k}=\uparrow \downarrow$ and $\{\sigma\}$ is a multi-index describing an arbitrary set of the lattice spins. The vacancy state $|0\rangle_{i}$ is a total spin singlet defined above.

The ground state at $J=0$ is degenerate with respect to spin. We can therefore choose a FM spin configuration. An arbitrary one-hole state is then given by

$$
|\Phi\rangle=\sum_{i} \phi_{i}|i,\{\uparrow\}\rangle
$$

The energy of such a state is given by

$$
\sum_{i j}\left\langle\Phi\left|H_{J=0}\right| \Phi\right\rangle=-\sum_{i j} t_{i j} \bar{\phi}_{j} \phi_{i}
$$

The corresponding Schrödinger equation reads

$$
\sum_{j}\left(t_{i j}-E \delta_{i j}\right) \phi_{j}=0
$$

The lowest-energy solution for the nearest-neighbor (nn) interaction reads

$$
\phi_{j}=1 / \sqrt{N_{s}}, \quad E_{0}=-2 t,
$$

with $N_{s}$ being the total number of the lattice sites.

To consider a state with $N$ holes one should generalize Eq. (A4) to include $N$ fermionic (hole) states:

$$
\left|\Phi_{N}\right\rangle=\sum_{i_{1}, i_{2}, \ldots, i_{N}} \phi_{i_{1}, i_{2}, \ldots, i_{N}}\left|i_{1}, i_{2}, \ldots, i_{N}\{\uparrow\}\right\rangle,
$$

where the function $\phi_{i_{1}, i_{2}, \ldots, i_{N}}$ is antisymmetric with respect to the index permutation. A corresponding Schrödinger $N$-particle equation can be then written out explicitly. Alternatively, one can quantize Eq. (A6) with exactly the same effect. Namely, the $c$-valued amplitudes $\phi_{i}$ are replaced by the fermion operators

$$
\phi_{i} \rightarrow \hat{\phi}_{i}=: f_{i}, \quad\left[f_{i}^{+}, f_{j}\right]_{+}=\delta_{i j} .
$$

The $N$-hole generalization of the Hamiltonian $H_{0}$ then reads

$$
H_{0}=-\sum_{i j} t_{i j} f_{i}^{+} f_{j}, \quad \sum_{i} f_{i}^{\dagger} f_{i}=N .
$$

This Hamiltonian describes spinless fermions hopping in a $1 \mathrm{D}$ lattice. In the case of the nn interaction, the ground-state energy becomes

$$
E_{0}=-\frac{2 t}{\pi} \sin (\pi \delta), \quad \delta=\frac{N}{N_{s}}=\frac{1}{N_{s}}\left\langle\sum_{i} f_{i}^{+} f_{i}\right\rangle_{H_{0}} .
$$

The spin degeneracy is lifted by the effective spin-spin interaction:

$$
H_{t-J}^{g r}=-\frac{2 t}{\pi} \sin (\pi \delta)+J_{\mathrm{eff}} \sum_{i j} \vec{S}_{i} \cdot \vec{S}_{j}+O\left(J^{2}\right), \quad J \rightarrow 0
$$

We have

$$
\begin{aligned}
J_{\mathrm{eff}} & =J\left\langle\left(1-f_{i}^{\dagger} f_{i}\right)\left(1-f_{j}^{\dagger} f_{j}\right)\right\rangle_{H_{0}} \\
& =J\left((1-\delta)^{2}-\frac{\sin ^{2} \pi(1-\delta)}{\pi^{2}}\right) .
\end{aligned}
$$

In terms of the electron density $n_{e}=1-\delta$, Eq. (A8) becomes

$$
\begin{aligned}
H_{t-J}^{g r}= & -\frac{2 t}{\pi} \sin \left(\pi n_{e}\right) \\
& +J\left(n_{e}^{2}-\frac{\sin ^{2} \pi n_{e}}{\pi^{2}}\right) \sum_{i j} \vec{S}_{i} \cdot \vec{S}_{j}+O\left(J^{2}\right),
\end{aligned}
$$

which agrees with the Bethe ansatz result obtained for the canonical $t$ - $J$ model given by Eq. (1) [12].
[1] See, e.g., P. A. Lee, N. Nagaosa, and X.-G. Wen, Rev. Mod. Phys. 78, 17 (2006), and references therein.

[2] A. Ferraz, E. Kochetov, and B. Uchoa, Phys. Rev. Lett. 98, 069701 (2007); R. T. Pepino, A. Ferraz, and E. Kochetov, Phys. Rev. B 77, 035130 (2008).
[3] M. M. Maska, M. Mierzejewski, A. Ferraz, and E. A. Kochetov, J. Phys. Condens. Matter 21, 045703 (2009); M. M. Maska, M. Mierzejewski, and E. A. Kochetov, Philos. Mag. 95, 583 (2015).

[4] T. C. Ribeiro and X.-G. Wen, Phys. Rev. Lett. 95, 057001 (2005); Phys. Rev. B 74, 155113 (2006). 
[5] A. Ferraz and E. Kochetov, Eur. Phys. J. B 86, 512 (2013).

[6] N. V. Prokofev, B. V. Svistunov, and I. S. Tupitsyn, Phys. Lett. A 238, 253 (1998).

[7] N. V. Prokof'ev, B. V. Svistunov, and I. S. Tupitsyn, J. Exp. Theor. Phys. 87, 310 (1998).

[8] N. Metropolis et al., J. Chem. Phys. 21, 1087 (1953).
[9] M. Troyer, F. Alet, S. Trebst, and S. Wessel, AIP Conf. Proc. 690, 156 (2003).

[10] Ar. Abanov, A. V. Chubukov, and J. Schmalian, Adv. Phys. 52, 119 (2003).

[11] E. H. da Silva Neto et al., Science 343, 393 (2014).

[12] H. Shiba and M. Ogata, Int. J. Mod. Phys. B 5, 31 (1991). 